\title{
Assessment and validation of proposed classification tools for brainstem cavernous malformations
}

\author{
*Alejandro N. Santos, MD,1 Laurèl Rauschenbach, MD,' Marvin Darkwah Oppong, MD, ${ }^{1}$ \\ Bixia Chen, MD, ${ }^{1}$ Annika Herten, MD, ${ }^{1}$ Michael Forsting, MD, ${ }^{2}$ Ulrich Sure, MD, ${ }^{1}$ and \\ Philipp Dammann, MD'
}

\begin{abstract}
1Department of Neurosurgery and Spine Surgery, University Hospital Essen; and 2Institute of Diagnostic and Interventional Radiology and Neuroradiology, University Hospital Essen, Essen, Germany
\end{abstract}

\begin{abstract}
OBJECTIVE Treatment indications for patients with brainstem cavernous malformations (BSCMs) remain difficult and controversial. Some authors have tried to establish classification tools to identify eligible candidates for surgery. Authors of this study aimed to validate the performance and replicability of two proposed BSCM grading systems, the LawtonGarcia (LG) and the Dammann-Sure (DS) systems.

METHODS For this cross-sectional study, a database was screened for patients with BSCM treated surgically between 2003 and 2019 in the authors' department. Complete clinical records, preoperative contrast-enhanced MRI, and a postoperative follow-up $\geq 6$ months were mandatory for study inclusion. The modified Rankin Scale (mRS) score was determined to quantify neurological function and outcome. Three observers independently determined the LG and the DS score for each patient.
\end{abstract}

RESULTS A total of 67 patients met selection criteria. Univariate and multivariate analyses identified multiple bleedings $(p=0.02$, OR 5.59), lesion diameter (> $20 \mathrm{~mm}, p=0.007$, OR 5.43), and patient age (> 50 years, $p=0.019$, OR 4.26) as predictors of an unfavorable postoperative functional outcome. Both the LG (AUC $=0.72, p=0.01)$ and the DS (AUC = $0.78, p<0.01)$ scores were robust tools to estimate patient outcome. Subgroup analyses confirmed this observation for both grading systems (LG: $p=0.005$, OR 6; DS: $p=0.026$, OR 4.5), but the combined use of the two scales enhanced the test performance significantly $(p=0.001$, OR 22.5).

CONCLUSIONS Currently available classification systems are appropriate tools to estimate the neurological outcome after BSCM surgery. Future studies are needed to design an advanced scoring system, incorporating items from the LG and the DS score systems.

https://thejns.org/doi/abs/10.3171/2020.6.JNS201585

KEYWORDS brainstem CCM; cerebral cavernous malformation; CCM surgery; Dammann-Sure grading system; Lawton-Garcia grading system; vascular disorders

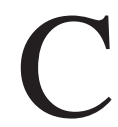
EREBRAL cavernous malformations (CCMs) are abnormal low-flow vascular sinusoidal endothelial cell caverns within the central nervous system ${ }^{1}$ and are considered the second most common type of neurovascular malformation. ${ }^{2}$ They occur in either sporadic or familial form. ${ }^{3}$ Oftentimes, CCMs are diagnosed incidentally and tend to have a benign natural history, usually requiring only clinical and radiological follow-up. ${ }^{4,5}$ However, a considerable number of CCMs become symptomatic with new-onset headache, cavernoma-related epilepsy, or focal neurological deficits, mainly because of their ten- dency to cause hemorrhage. ${ }^{5}$ Such events have been defined as "symptomatic hemorrhages." Depending on the severity of clinical symptoms, lesion localization, history of symptomatic bleeding, and lesion size, surgical removal of the CCM may be indicated. ${ }^{7-9}$ Notably, 20\%-35\% of CCMs are located within the brainstem.5,10,11 Brainstem cavernous malformations (BSCMs) represent a unique subgroup of CCMs, as they can cause considerable functional impairment due to an increased risk of symptomatic hemorrhage ${ }^{3,12}$ compared to that with otherwise localized CCMs. ${ }^{5,13}$ Although technically challenging and associ-

ABBREVIATIONS AUC = area under the curve; $\mathrm{BSCB}=$ brainstem cavernoma bleeding; $\mathrm{BSCM}=$ brainstem cavernous malformation; $\mathrm{CCM}=$ cerebral cavernous malformation; DS = Dammann-Sure; DVA = developmental venous anomaly; LG = Lawton-Garcia; $m R S=$ modified Rankin Scale; ROC = receiver operating characteristic. SUBMITTED May 1, 2020. ACCEPTED June 16, 2020.

INCLUDE WHEN CITING Published online October 16, 2020; DOI: 10.3171/2020.6.JNS201585.

${ }^{*}$ A.N.S. and L.R. contributed equally to this work. 
ated with significant perioperative morbidity, ${ }^{11}$ surgical treatment of BSCM is a well-established procedure. ${ }^{11,14-16}$ Because of the complex brainstem anatomy, treatment decisions for patients with BSCM remain difficult and controversial.9 Only a few authors have tried to establish CCM grading systems to enable the identification of good candidates for surgery and to predict their individual outcomes. Up to now, three different grading systems have been proposed, one of them established by our own group..$^{17-19}$ Two of these scales are specifically applicable to BSCM, that is, the Lawton-Garcia (LG) grading system and the Dammann-Sure (DS) classification.

In order to include such grading systems into the clinical routine, repetitive validation is necessary but has not been sufficiently performed yet. Therefore, with this study, we aimed to validate the performance of these two available BSCM grading systems using a single center cohort of patients. In addition, we evaluated the interobserver agreement of the scales to test their clinical applicability. Finally, we examined whether a combination of the two grading systems would provide increased predictive performance.

\section{Methods \\ Data Collection}

The study was conducted at our tertiary care hospital in accordance with all guidelines set forth by the approving institutional review board. We performed a crosssectional study of all patients admitted to our department from January 1, 2003, until June 30, 2019, who fulfilled the inclusion criteria listed below. Clinical data, preoperative multiplanar contrast-enhanced MRI, and functional outcome with pre- and postoperative modified Rankin Scale (mRS) scores were obtained for each patient. The LG and DS scores (see below) were calculated for each patient. The study was conducted according to the Declaration of Helsinki, and local ethics approval was obtained. Informed consent was obtained from all participants. The study was performed according to the STROBE protocol.

\section{Inclusion Criteria and Exclusion Criteria}

Patients aged $\geq 18$ years with BSCM surgically treated at our center were included. All patients needed to have a minimum of 6 months of postoperative follow-up. Patients without a complete clinical assessment or missing imaging data were excluded from the study.

\section{Grading Systems}

The LG scale is based on clinical and MRI-derived items, that is, lesion size, lesion crossing of the axial midpoint, presence of a developmental venous anomaly (DVA), patient age, and hemorrhage timing. ${ }^{18}$ Each item can be assigned points from 0 to 2, depending on the item, and the final score is the sum of the points for all the items. The total points range from 0 to 7. A favorable outcome ( $\mathrm{mRS}$ score $\leq 2)$ is indicated by low grades (LG grades 0 III) and an unfavorable outcome ( $\mathrm{mRS}>2)$ by high grades (LG grades IV-VII).

The DS scale is based only on MRI-derived items. ${ }^{19}$ The classification system was initially established to rate the difficulty of surgical dissection of a CCM in eloquent areas. It was validated with postoperative diffusionweighted MRI, a surgical questionnaire, and functional outcome. Defining features are the presence of macrocaverns, lesion shape, form of hemosiderin deposits, and contrast enhancement, whereas associated features are a DVA, a macro-hemorrhage, perifocal edema, and a multiaged macro-hemorrhage. The combination or quality of items applies to one type, and the typing ranges from 1 to 3. In cases of acute hemorrhage, the suffix "a" is additionally applied; otherwise, the suffix " $b$ " is used. Favorable resection is indicated by low types (types $1 \mathrm{a} / \mathrm{b}$ and $2 \mathrm{a} / \mathrm{b}$ ), and difficult resection is indicated by high types (types $3 a / b)$.

\section{Imaging Analysis}

Imaging data were independently analyzed by three investigators (A.N.S., M.D.O., P.D.), including score determination for each patient. In cases of total disagreement, the most experienced investigator (P.D.) decided. Interrater agreement was indexed using Fleiss' kappa statistic. Kappa represents the strength of agreement above the level of chance according to the following rating: ${ }^{20} 0.2-0.4$, slight to fair; 0.41-0.6, moderate; 0.61-0.8, substantial; > 0.8 , excellent.

\section{Statistical Analysis}

We used SPSS 22 (IBM Corp.) for all statistical analyses. Univariate analyses were performed to determine predictors of postoperative clinical outcome. For dichotomized variables, the chi-square test (sample size $>5$ ) or Fisher exact test (sample size $\leq 5$ ) was used. Continuous variables were tested with the Student t-test (normally distributed data) or Mann-Whitney U-test (nonnormally distributed data). Data distribution was determined with the Kolmogorov-Smirnov test. Multivariate analyses were performed with a binary regression model and were based on an a priori hypothesis that predictors of unfavorable outcomes were analogous to those found in the LG grading system ${ }^{18}$ including patient age, presence of a DVA, chronic hemorrhage, lesion size, and lesion crossing the brainstem midpoint. As stated in the literature, ${ }^{18}$ favorable surgical outcomes (mRS score $\leq 2$ ) versus unfavorable ones (mRS score $>2$ ) were considered at the last clinical evaluation adjusted for the follow-up observation time of a minimum of 6 months. To evaluate the prognostic performance of the different grading systems, the receiver operating characteristic (ROC) metric was used, quantified by the area under the curve (AUC), with values close to 1 indicating a perfect score and values close to 0.5 reflecting a nonrelevant score. A more detailed classification was applied according to the following rating: ${ }^{21} 0.7-0.8$, acceptable; $0.8-0.9$, excellent; $>0.9$, outstanding. A univariate analysis of patient subpopulations according to both LG and DS system scores was performed with the dependent outcome variable favorable $(\mathrm{mRS} \leq 2)$ versus unfavorable $(\mathrm{mRS}>2)$ at the last clinical evaluation. Scores in each grading system were dichotomized as stated in each of the systems. A p value $<0.05$ was defined as statistically significant. 


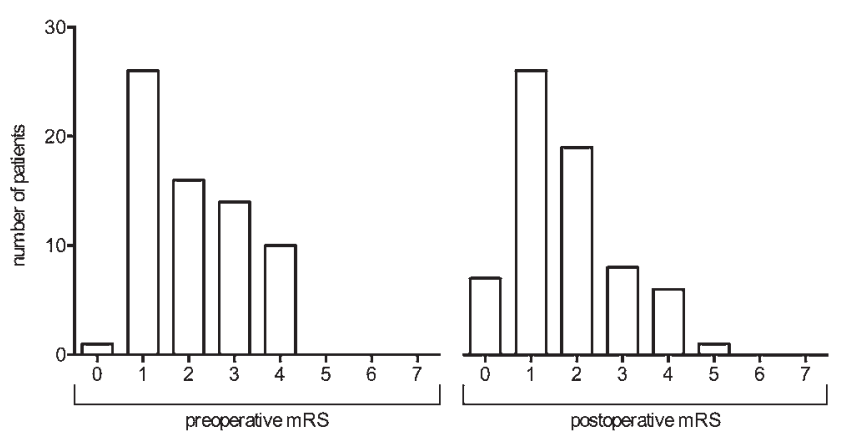

FIG. 1. Distribution of mRS scores preoperatively on admission and postoperatively at the final follow-up.

\section{Results}

\section{Patient Demographics and Outcomes}

Eighty-nine potentially eligible patients were identified. In 19 patients, the clinical data set was incomplete, and 4 patients were lost to follow-up. Therefore, 22 patients were excluded from the study and 67 patients were considered for analysis. The mean age was $40.6 \pm 12.7$ years, and 41 individuals $(61.2 \%)$ were female. A total of 20 patients $(30.0 \%)$ had a DVA associated with the BSCM, and $33(49.3 \%)$ of the lesions crossed the axial midline. Thirty-two patients (47.8\%) suffered an acute hemorrhage, $18(26.9 \%)$ revealed subacute hemorrhage, and 17 (25.4\%) had chronic hemorrhage, according to the definition of Garcia and colleagues. ${ }^{18}$ Almost half of all the patients (47.8\%) experienced more than one bleeding. The BSCMs were rather heterogeneously distributed within the brainstem. The most common location was the pons, accounting for 25 cases (37.3\%), followed by the mesencephalon in 14 cases $(20.9 \%)$. The mean diameter of the brainstem cavernoma bleeding (BSCB) was $18.5 \pm 7.7 \mathrm{~mm}$. On admission, 43 patients $(64.2 \%)$ were in good clinical condition (mRS score $\leq 2)$. A favorable outcome after cavernoma resection was observed in 52 patients $(77.6 \%)$, and the majority of patients $(79.1 \%)$ revealed improved or unchanged scores. On the contrary, an unfavorable outcome (mRS score $>2$ ) was found in 15 patients $(22.4 \%), 8$ (11.9\%) whose condition had worsened compared to their preoperative baseline (Fig. 1 and Table 1).

\section{Predictors of Postoperative Outcome}

Univariate analysis identified preoperative multiple bleedings (OR 5.59, 95\% CI 1.39-22.44, $\mathrm{p}=0.02$ ) as the only statistically significant predictor of an unfavorable postoperative outcome. Midline crossing (OR 2.18, 95\% CI $0.64-7.37, \mathrm{p}=0.24)$ and the presence of a DVA (OR $1.41,95 \%$ CI $0.41-4.9, \mathrm{p}=0.74$ ) showed no statistically significant influence on outcome (Table 1). Multivariate analysis identified a maximum BSCB diameter $>20 \mathrm{~mm}$ (OR 5.43, 95\% CI 1.58-18.69, $\mathrm{p}=0.007$ ) and patient age $>50$ years (OR 4.26, 95\% CI 1.27-14.33, $\mathrm{p}=0.019)$ as independent predictors of an unfavorable postoperative outcome, whereas midline crossing (OR 3.21, 95\% CI $0.9-11.4, \mathrm{p}=0.07$ ), DVA (OR 0.82, 95\% CI 0.23-2.96, $\mathrm{p}=$ 0.76 ), and chronic hemorrhage (> 8 weeks; OR 1.67, $95 \%$
CI $0.48-5.83, \mathrm{p}=0.42$ ) were not independent predictors (Table 2).

\section{Performance of Proposed BSCM Grading Systems}

We performed an ROC analysis with AUC metrics and demonstrated that the LG grade (AUC $=0.72,95 \%$ CI $0.56-0.88, \mathrm{p}=0.01$ ) and the DS score (AUC $=0.78,95 \%$ CI $0.64-0.9, \mathrm{p}<0.01 ; \mathrm{AUC}=0.74,95 \%$ CI $0.62-0.87, \mathrm{p}<$ 0.01 ) significantly correlated with postoperative outcome. The predictive value of each score was considered as substantial (AUC 0.8-0.7; Fig. 2 and Table 3). Subsequent analysis revealed that $75 \%$ of all patients with a favorable postoperative outcome were correctly classified in both grading systems (LG grades $0-$ III, DS types 1-2). An unfavorable outcome (LG grades IV-VII, DS type 3) was correctly predicted in $66.7 \%$ and $60 \%$ of patients, respectively (Table 4). Both the LG (OR 6, 95\% CI 1.73-20.81, $\mathrm{p}=0.005)$ and the DS (OR 4.5, 95\% CI 1.34-15.07, $\mathrm{p}=$ 0.026 ) grading systems were strong predictors of the postoperative functional outcome. The combined use of the two scales enhanced the predictive performance (OR 22.5, 95\% CI 3.33-152, $\mathrm{p}=0.001$ ).

\section{Interobserver Variability}

Independent investigators determined the individual scores for a total of 71 patients (A.N.S., M.D.O., P.D.). Evaluation revealed an interobserver disagreement in $25.4 \%$ (DS system) and 8.5\% (LG system) of all cases. According to Fleiss' kappa statistic, the DS grading system had substantial interobserver agreement $(\kappa=0.7)$, whereas the LG grading system revealed excellent agreement $(\kappa=$ 0.92; Supplemental Fig. 1).

\section{Discussion \\ Validation of Proposed Grading Systems}

Patient selection is mandatory for risk stratification and to identify eligible candidates for BSCM surgery. This selection can be performed by determining a wide variety of clinical and anatomical variables. Although they have limits in terms of sensitivity and specificity, grading systems can be helpful tools for neurosurgeons on which to base their clinical decisions, that is, using not only personal experience but also objective algorithms.

Currently, three grading systems for CCM are available. ${ }^{17-19}$ While two studies have focused on CCM in general, Garcia and colleagues have established a grading system specifically for BSCM..$^{18}$ The first study, published in 2011, classifies the majority of BSCMs as high-risk grade 3 lesions with an almost 50\% chance of long-term disability after surgery. ${ }^{17}$ The grading system of Dammann and colleagues differentiates three types of CCM (DS types 1-3) according to the preoperative MRI data, measuring variables such as size of the caverns, lesion shape, and deposits of hemosiderin. ${ }^{19}$ Moreover, the three-tiered classification system is extended to a more detailed grading system with six subgroups of CCM through the implementation of the item lesion-associated hemorrhage (DS types $1 \mathrm{a}-3 \mathrm{~b})$. Depending on the DS type, the difficulty of resection varies. The LG grading system was proposed in 2015 and combines clinical and imaging data to guide 
TABLE 1. Univariate analysis of predictors of an unfavorable functional postoperative outcome

\begin{tabular}{|c|c|c|c|c|c|}
\hline Variable & mRS Score Same or Improved & mRS Score Worse & p Value & OR & $95 \% \mathrm{Cl}$ \\
\hline No. of patients & $53(79.1 \%)$ & $14(20.9 \%)$ & & & \\
\hline Mean age in yrs & $40.3 \pm 13.1$ & $41.5 \pm 11.6$ & $0.76^{*}$ & NA & NA \\
\hline Female sex & $32(60.4 \%)$ & $9(64.3 \%)$ & $>0.99 \dagger$ & 1.18 & $0.35-4.12$ \\
\hline Lesion location & & & $0.71 \dagger$ & NA & NA \\
\hline Medullary & $6(11.3 \%)$ & $0(0 \%)$ & & & \\
\hline Pontomedullary & $9(17.0 \%)$ & $2(14.3 \%)$ & & & \\
\hline Pontine & $19(35.8 \%)$ & $6(42.9 \%)$ & & & \\
\hline Pontomesencephalic & $9(17.0 \%)$ & $2(14.3 \%)$ & & & \\
\hline Mesencephalic & $10(18.9 \%)$ & $4(28.6 \%)$ & & & \\
\hline Lesion side & & & $0.9+$ & NA & NA \\
\hline Lt & $26(49.1 \%)$ & $6(42.9 \%)$ & & & \\
\hline Rt & $24(45.3 \%)$ & $7(50 \%)$ & & & \\
\hline Medial & $3(5.7 \%)$ & $1(7.1 \%)$ & & & \\
\hline Mean max BSCB diameter in $\mathrm{mm}$ & $18.3 \pm 8.3$ & $19 \pm 5.6$ & $0.55^{*}$ & NA & NA \\
\hline DVA & $15(28.3 \%)$ & $5(35.7 \%)$ & $0.74 \dagger$ & 1.41 & $0.41-4.9$ \\
\hline Midline crossing & $24(45.3 \%)$ & $9(64.3 \%)$ & $0.24 \dagger$ & 2.18 & $0.64-7.37$ \\
\hline Preop multiple bleedings & $21(39.6 \%)$ & $11(78.6 \%)$ & $0.02 \dagger$ & 5.59 & $1.39-22.44$ \\
\hline Age of hemorrhage & & & $0.93 \dagger$ & NA & NA \\
\hline Acute & $26(49.1 \%)$ & $6(42.9 \%)$ & & & \\
\hline Subacute & $14(26.4 \%)$ & $4(28.6 \%)$ & & & \\
\hline Chronic & $13(24.5 \%)$ & $4(28.6 \%)$ & & & \\
\hline mRS score preop & & & $0.1 \dagger$ & NA & NA \\
\hline 0 & $1(1.9 \%)$ & $0(0 \%)$ & & & \\
\hline 1 & $20(37.7 \%)$ & $6(42.9 \%)$ & & & \\
\hline 2 & $14(26.4 \%)$ & $2(14.3 \%)$ & & & \\
\hline 3 & $8(15.1 \%)$ & $6(42.9 \%)$ & & & \\
\hline 4 & $10(18.9 \%)$ & $0(0 \%)$ & & & \\
\hline 5 & $0(0 \%)$ & $0(0 \%)$ & & & \\
\hline 6 & $0(0 \%)$ & $0(0 \%)$ & & & \\
\hline mRS score postop & & & $<0.001 \dagger$ & NA & NA \\
\hline 0 & $7(13.2 \%)$ & $0(0 \%)$ & & & \\
\hline 1 & $26(49.1 \%)$ & $0(0 \%)$ & & & \\
\hline 2 & $13(24.5 \%)$ & $6(42.9 \%)$ & & & \\
\hline 3 & $6(11.3 \%)$ & $2(14.3 \%)$ & & & \\
\hline 4 & $1(1.9 \%)$ & $5(35.7 \%)$ & & & \\
\hline 5 & $0(0 \%)$ & $1(7.1 \%)$ & & & \\
\hline 6 & $0(0 \%)$ & $0(0 \%)$ & & & \\
\hline
\end{tabular}

NA = not applicable.

Univariate analysis of demographic, clinical, and anatomical factors for association with functional postoperative outcome (changes in mRS score between admission and final follow-up). All patients with a final follow-up $\geq 6$ months after surgery were included. Values expressed as the mean \pm standard error or as number $(\%)$, unless indicated otherwise. Boldface type indicates statistical significance.

* Student's t-test or Mann-Whitney U-test.

†Chi-square test or Fisher exact test.

surgical decision-making and to predict the postoperative functional outcome..$^{18}$ This score takes into account five variables, such as lesion size, crossing of the axial midpoint, presence of an associated DVA, age of the patient, and time of hemorrhage, to build an eight-tiered classification system.

Up to now, only one study has investigated the performance of one proposed grading system. ${ }^{22}$ According to the authors of that study, there is a sufficient correlation not only between the LG grading scale and postoperative functional outcome, but also between the LG grading system and postoperative quality of life. Our study confirms that both grading systems are statistically significant predictors of postoperative outcome (Table 3 and Fig. 2). Since imaging data are mandatory for both classification tools, the determination of variables may differentiate between different raters. Thus, to ensure reliability, we additionally investigated the interobserver accuracy and found excellent agreement for the LG grade (Supplemental Fig. 1). This can be explained by the fact that the variables used for the LG tool, such as patient age, lesion size, or presence of DVA, are clear-cut variables that are easy to obtain. The 
TABLE 2. Multivariate analysis of predictors of an unfavorable functional postoperative outcome

\begin{tabular}{lccc}
\hline \multicolumn{1}{c}{ Variable } & $\mathrm{p}$ Value & OR & $95 \% \mathrm{Cl}$ \\
\hline Maximum BSCB diameter $>20 \mathrm{~mm}$ & $\mathbf{0 . 0 0 7}$ & 5.43 & $1.58-18.69$ \\
\hline Midline crossing & 0.071 & 3.21 & $0.9-11.4$ \\
\hline DVA & 0.76 & 0.82 & $0.23-2.96$ \\
\hline Age $>50$ yrs & $\mathbf{0 . 0 1 9}$ & 4.26 & $1.27-14.33$ \\
\hline Chronic hemorrhage $(>8$ wks) & 0.424 & 1.67 & $0.48-5.83$ \\
\hline
\end{tabular}

Multivariate binary logistic regression model with the dependent outcome variable as favorable ( $m R S \leq 2)$ versus unfavorable ( $m R S>2$ ) outcome at the last clinical evaluation. All patients with a last follow-up of $\geq 6$ months were included. Boldface type indicates statistical significance.

DS score only includes MRI-based items and thus determines more subjective variables, such as lesion shape. These variables are more prone to interpretation, resulting in a lower interobserver agreement rate. However, our data revealed substantial agreement for the DS tool, making the score also applicable to clinical use.

\section{Predictors of Postoperative Outcome}

As stated above, the LG grading system takes five variables into account. When assessed individually, none of these variables was a significant predictor of postoperative outcome. Interestingly, multiple bleedings, which is not considered in this scale, was the only significant predictor of outcome in our analysis. Notably, this variable is elaborated on in the paper by Dammann and colleagues, who showed that evidence of several bleeding events on MRI correlated with a more difficult surgical dissection of the BSCM because "multiage bleeding" gives the lesion different consistencies. ${ }^{19}$

In our multivariate analysis, we investigated all items of the LG grading system. In our patient population, a BSCB diameter $>20 \mathrm{~mm}$ and an age $>50$ years were independent predictors of an unfavorable postoperative outcome (Table 2). A young age is associated with a stronger resistance to surgery, improved recovery from surgery, and less comorbidity, making it an obvious predictor of a favorable outcome. The same applies to lesion size, since large lesions can involve more eloquent brainstem areas, making the resection more difficult.

Even though both grading systems consider chronic hemorrhage as a predictor of a favorable outcome, this item was not an independent predictor in our multivariate analysis. The same applied to DVA and axial midline crossing. Both study cohorts (from Garcia et al..$^{18}$ and Dammann et al. ${ }^{19}$ ) reveal a limited number of patients, which could be one explanation for the discrepancy observed here.

\section{Combined Use of Proposed Grading Systems}

In our study, we demonstrated that both classification systems are reliable tools to predict postoperative functional outcome (Table 4). While the LG grade includes clinical and radiological data, the DS type is mainly focused on imaging data, which raises the question of whether the combined use of the two scores might enhance the predictive performance. Notably, we found that the com-

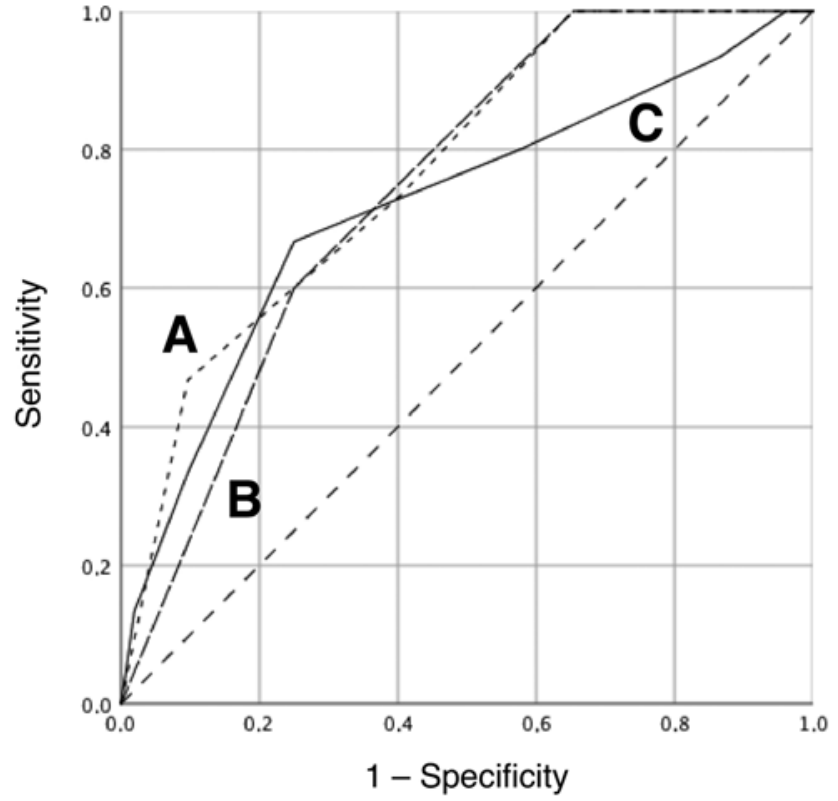

FIG. 2. ROC analysis to illustrate the test performance of available BSCM grading systems. DS score with subtypes for acute hemorrhage (A), DS score without subtypes for acute hemorrhage (B), and LG grade (C)

bination of the two grading scales is a stronger predictor for postoperative outcome, with an OR of 22.5, compared to 6 (LG alone) and 4.5 (DS alone). This is a new finding and requires further investigation in future BSCM studies.

\section{External Validity}

Compared to those in meta-analyses of BSCM trials, our cohort seems representative in terms of patient characteristics and postoperative outcome. ${ }^{11,23}$ This observation increases the external validity of our reported results.

\section{Study Limitations}

CCM is a rare vascular disease, accounting for $10 \%-$ $15 \%$ of all intracranial vascular malformations. ${ }^{2}$ Since only $35 \%$ of these lesions are localized in the brainstem ${ }^{10}$ and only a small percentage of these lesions undergo surgical removal, single-center trials with large sample sizes remain difficult. Additionally, as BSCM is a rather rare

TABLE 3. Performance of proposed BSCM grading systems

\begin{tabular}{cccc}
\hline Scale & AUC & p Value & $95 \% \mathrm{Cl}$ \\
\hline LG (grade 0-VII) & 0.72 & 0.01 & $0.56-0.88$ \\
\hline DS (type 1a-3b) & 0.78 & $<0.01$ & $0.64-0.9$ \\
\hline DS (type 1-3) & 0.74 & $<0.01$ & $0.62-0.87$ \\
\hline
\end{tabular}

ROC analysis with AUC metrics to determine the test performance of available BSCM grading systems. Functional postoperative outcome (mRS $\leq 2$ vs $m R S$ $>2$ ) at the last clinical evaluation was the dependent variable. All patients with a last follow-up $\geq 6$ months were included. An AUC $>0.7$ is considered as an acceptable test performance. The $p$ values represent the asymptotic significance. Boldface type indicates statistical significance. 
TABLE 4. Functional postoperative outcome according to proposed BSCM grading systems

\begin{tabular}{|c|c|c|c|c|c|}
\hline Variable & $m R S \leq 2$ & $m R S>2$ & $p$ Value & OR & $95 \% \mathrm{Cl}$ \\
\hline No. of patients & $52(77.6 \%)$ & $15(22.4 \%)$ & & & \\
\hline LG grade & & & $0.005^{*}$ & 6 & $1.73-20.81$ \\
\hline $0-111$ & $39(75 \%)$ & $5(33.3 \%)$ & & & \\
\hline IV-VII & $13(25 \%)$ & $10(66.7 \%)$ & & & \\
\hline DS type & & & $0.026^{*}$ & 4.5 & $1.34-15.07$ \\
\hline $1 a / b, 2 a / b$ & $39(75 \%)$ & $6(40 \%)$ & & & \\
\hline $3 a / b$ & $13(25 \%)$ & $9(60 \%)$ & & & \\
\hline Combination $†$ & & & $0.001^{*}$ & 22.5 & $3.33-152$ \\
\hline LG grade 0 -III \& DS types $1 \mathrm{a} / \mathrm{b}$ \& 2a/b & $30(88.2 \%)$ & $2(25 \%)$ & & & \\
\hline LG grades IV-VII \& DS type $3 a / b$ & $4(11.8 \%)$ & $6(75 \%)$ & & & \\
\hline
\end{tabular}

vascular disease with few cases, our results are mainly representative of experienced, high-volume centers. Moreover, our data were in part obtained retrospectively, which can lead to well-known information and selection biases. Since the data set in this study is limited to surgically treated patients, our results do not provide guidance on conservatively treated BSCM. In a former study, a total of 21 patients from our current cohort has already been used to establish the DS score. This might bias the data of our current investigation. Nevertheless, our study contributes novel data and proposes the combined use of existing classification systems. Prospective multicenter trials are needed to validate our assumption and to examine new predictors of patient outcome.

\section{Conclusions}

The LG and the DS classification tools are appropriate for estimating the neurological outcome after BSCM surgery. This study confirms the predictive accuracy of both grading systems and gives evidence supporting their combined use.

\section{References}

1. Batra S, Lin D, Recinos PF, et al. Cavernous malformations: natural history, diagnosis and treatment. Nat Rev Neurol. 2009;5(12):659-670.

2. Taslimi S, Modabbernia A, Amin-Hanjani S, et al. Natural history of cavernous malformation: systematic review and meta-analysis of 25 studies. Neurology. 2016;86(21):19841991.

3. Gross BA, Lin N, Du R, Day AL. The natural history of intracranial cavernous malformations. Neurosurg Focus. 2011; 30(6):E24.

4. Moore SA, Brown RD Jr, Christianson TJH, Flemming KD. Long-term natural history of incidentally discovered cavernous malformations in a single-center cohort. J Neurosurg. 2014;120(5):1188-1192.

5. Horne MA, Flemming KD, Su IC, et al. Clinical course of untreated cerebral cavernous malformations: a meta-analysis of individual patient data. Lancet Neurol. 2016;15(2):166-173.

6. Al-Shahi Salman R, Berg MJ, Morrison L, Awad IA. Hemor- rhage from cavernous malformations of the brain: definition and reporting standards. Stroke. 2008;39(12):3222-3230.

7. Giliberto G, Lanzino DJ, Diehn FE, et al. Brainstem cavernous malformations: anatomical, clinical, and surgical considerations. Neurosurg Focus. 2010;29(3):E9.

8. Rinkel LA, Al-Shahi Salman R, Rinkel GJE, Greving JP. Radiosurgical, neurosurgical, or no intervention for cerebral cavernous malformations: a decision analysis. Int J Stroke. 2019;14(9):939-945.

9. Akers A, Al-Shahi Salman R, Awad IA, et al. Synopsis of guidelines for the clinical management of cerebral cavernous malformations: consensus recommendations based on systematic literature review by the Angioma Alliance Scientific Advisory Board Clinical Experts Panel. Neurosurgery. 2017; 80(5):665-680.

10. Kupersmith MJ, Kalish H, Epstein F, et al. Natural history of brainstem cavernous malformations. Neurosurgery. 2001; 48(1):47-54.

11. Gross BA, Batjer HH, Awad IA, et al. Brainstem cavernous malformations: 1390 surgical cases from the literature. World Neurosurg. 2013;80(1-2):89-93.

12. Li D, Wu Z-Y, Liu P-P, et al. Natural history of brainstem cavernous malformations: prospective hemorrhage rate and adverse factors in a consecutive prospective cohort. J Neurosurg. Published online March 13, 2020. doi:10.3171/2019.12. JNS192856

13. Dammann P, Jabbarli R, Wittek P, et al. Solitary sporadic cerebral cavernous malformations: risk factors of first or recurrent symptomatic hemorrhage and associated functional impairment. World Neurosurg. 2016;91:73-80.

14. Abla AA, Turner JD, Mitha AP, et al. Surgical approaches to brainstem cavernous malformations. Neurosurg Focus. 2010; 29(3):E8.

15. Bertalanffy H, Benes L, Miyazawa T, et al. Cerebral cavernomas in the adult. Review of the literature and analysis of 72 surgically treated patients. Neurosurg Rev. 2002;25(1-2):1-55.

16. Ohue S, Fukushima T, Kumon Y, et al. Surgical management of brainstem cavernomas: selection of approaches and microsurgical techniques. Neurosurg Rev. 2010;33(3):315-324.

17. Kivelev J, Laakso A, Niemelä M, Hernesniemi J. A proposed grading system of brain and spinal cavernomas. Neurosurgery. 2011;69(4):807-814.

18. Garcia RM, Ivan ME, Lawton MT. Brainstem cavernous malformations: surgical results in 104 patients and a proposed grading system to predict neurological outcomes. Neurosurgery. 2015;76(3):265-278. 
19. Dammann P, Wrede K, Jabbarli R, et al. Of bubbles and layers: which cerebral cavernous malformations are most difficult to dissect from surrounding eloquent brain tissue? Neurosurgery. 2017;81(3):498-503.

20. Landis JR, Koch GG. The measurement of observer agreement for categorical data. Biometrics. 1977;33(1):159-174.

21. Mandrekar JN. Receiver operating characteristic curve in diagnostic test assessment. J Thorac Oncol. 2010;5(9):13151316.

22. Lashkarivand A, Ringstad G, Eide PK. Surgery for brainstem cavernous malformations: association between preoperative grade and postoperative quality of life. Oper Neurosurg (Hagerstown). 2020;18(6):590-598.

23. Kearns KN, Chen CJ, Tvrdik P, et al. Outcomes of surgery for brainstem cavernous malformations: a systematic review. Stroke. 2019;50(10):2964-2966.

\section{Disclosures}

The authors report no conflict of interest concerning the materials or methods used in this study or the findings specified in this paper.

\section{Author Contributions}

Conception and design: Dammann. Acquisition of data: Santos, Forsting. Analysis and interpretation of data: Rauschenbach, Darkwah Oppong, Dammann. Drafting the article: Santos,
Rauschenbach. Critically revising the article: Santos, Rauschenbach, Darkwah Oppong, Chen, Herten, Sure, Dammann. Reviewed submitted version of manuscript: Santos. Statistical analysis: Rauschenbach, Dammann. Administrative/technical/ material support: Rauschenbach, Darkwah Oppong, Sure, Dammann. Study supervision: Sure, Dammann.

\section{Supplemental Information}

Online-Only Content

Supplemental material is available with the online version of the article.

Supplemental Fig. 1. https://thejns.org/doi/suppl/10.3171/ 2020.6.JNS201585.

\section{Correspondence}

Alejandro N. Santos: University Hospital Essen, Germany. alejandro.santos@uk-essen.de. 